\title{
Testing the Ability of the Surprisingly Popular Method to Predict NFL Games
}

\author{
Michael D. Lee, Irina Danileiko, Julie Vi \\ Department of Cognitive Sciences \\ University of California, Irvine
}

\begin{abstract}
We consider the recently-developed "surprisingly popular" method for aggregating decisions across a group of people (Prelec, Seung, \& McCoy, 2017). The method has shown impressive performance in a range of decision-making situations, but typically for situations in which the correct answer is already established. We consider the ability of the surprisingly popular method to make predictions, in a situation where the correct answer does not exist at the time people are asked to make decisions. Specifically, we tested its ability to predict the winners of the 256 US National Football League (NFL) games in the 2017-2018 season. Each of these predictions used participants who self-rated as "extremely knowledgeable" about the NFL, drawn from a set of 100 participants recruited through Amazon Mechanical Turk (AMT). We compare the accuracy and calibration of the surprisingly popular method to a variety of alternatives: the mode and confidence-weighted predictions of the expert AMT participants, the individual and aggregated predictions of media experts, and a statistical Elo method based on the performance histories of the NFL teams. We find that the surprisingly popular method outperforms all of these alternatives, and has reasonable calibration properties relating the confidence of predictions to accuracy.
\end{abstract}

Keywords: surprisingly popular algorithm, wisdom the the crowd, sporting predictions, expertise, majority rule 


\section{Introduction}

Prelec et al. (2017) recently proposed the "surprisingly popular" method for aggregating multiple-choice decisions over a group of people. The method is motivated by the challenge of finding an accurate answer to a single question, especially in the situation where many people in the group could believe in the wrong answer. For example, if asked whether Seattle is the capital city of the state of Washington, many people mistakenly answer "yes". The key feature of the surprisingly popular method is that, as well as providing their answer, people are asked to estimate what percentage of other people they expect will also give the same answer. Thus, if somebody knows the capital of Washington is Olympia, but also realizes that most people mistakenly believe it is Seattle, both of these pieces of information can be expressed.

The surprisingly popular method combines the cognitive judgment (the basic decision) and the meta-cognitive judgment (the estimate of the decisions of others) by comparing the expected and observed proportions of people making a decision. The observed proportion is simply how many say "yes" to Seattle being the capital. The expected proportion combines the estimated percentages for those who say both "yes" and "no". A person who says "yes" and expects $90 \%$ of people to agree contributes to the expected proportion in the same way as person who says "no" but expects only $10 \%$ to agree. The final decision made by the surprisingly popular method compares the observed and expected proportions, and chooses the answer that has more observed agreement than is expected (i.e., the answer that is "surprisingly popular"). Intuitively, people who believe Seattle is the capital of Washington will tend to believe others will say the same, while those who know it is not will expect others to disagree. Thus, the expected agreement is very high, but the observed agreement is lower, because some knowledgeable people say "no". This means "no" will be the surprisingly popular answer.

Figure 1 provides a concrete demonstration of the operation of the surprisingly popular method, based on data for the Seattle question reported by Prelec et al. (2017). The two bar graphs show the distribution of meta-cognitive judgments. The distribution of estimated agreement from participants answering that Seattle is the capital of Washington is shown by the upper (blue) distribution, while the distri-

bution of estimated agreement from participants answering that Seattle is not the 


\section{Is Seattle the capital of Washington?}

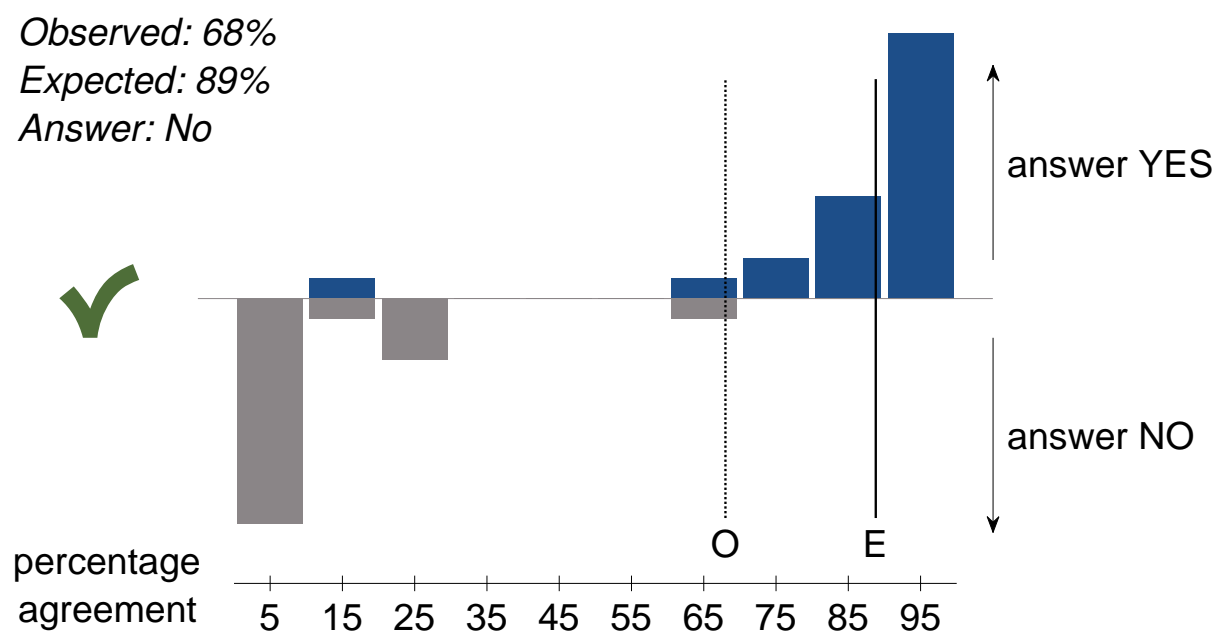

Figure 1. An example of the surprisingly popular method choosing the correct minority answer for the question "Is Seattle the capital of Washington?"

capital of Washington is shown by the lower (gray) distribution. Each bar in these distributions correspond to a $10 \%$ range in the meta-cognitive estimates so that, for example, the large downward gray bar at the far left indicates that many people answered that Seattle is not the capital of Washington and expected fewer than $10 \%$ of people to agree with their answer. The expected "E" and observed "O" levels of agreement calculated from these estimates are listed, and shown by solid and broken lines respectively. A majority of $68 \%$ of people answered that Seattle was the capital, but the expected agreement was $89 \%$, leading the surprisingly popular method to give the correct answer that Seattle is not the capital of Washington. The key contribution to the relatively high expected value is evident from the distributions of meta-cognitive judgments. A significant number of people who answered that Seattle is not the capital expected that few others would agree with them. Intuitively, these people know the correct minority answer, and their meta-cognitive estimates allow the surprisingly popular method to side with the minority.

Prelec et al. (2017) evaluate the accuracy of the surprisingly popular method in a number of domains, including trivia and general knowledge questions, medical diagnoses, and art price category evaluations. For all of these domains, the surprisingly popular method achieves impressive levels of accuracy, outperforming standard alter- 
natives like the majority answer, and the answer in which people express the greatest overall confidence. While the trivia and general knowledge domains have limited real-world applicability — outside the confines of a trivia competition, it is possible simply to look up the answers - the medical diagnosis and art evaluation domains clearly could have useful application. In both cases, an accurate decision based on cheap and simple behavioral judgments is a useful capability, since determining the true answer involves expensive medical testing in the first case, and time-consuming and complicated mechanisms like art auctions in the second case.

Perhaps the most interesting and important potential application of the surprisingly popular method, however, is to situations requiring genuine prediction, such as geopolitical forecasting or sporting predictions (Silver, 2012; Tetlock \& Gardner, 2016), where the true answer is in principle not knowable at the time people make decisions. None of the domains considered by Prelec et al. (2017) are of this type, unless the argument is made that the value of art does not exist until it is socially constructed by an auction or some other valuation mechanism. Given this gap in evaluation, our goal is to provide a direct predictive test of the surprisingly popular method, by evaluating its performance forecasting the outcome of the 256 games played in the regular season of the US National Football League (NFL) in the 20172018 season.

The structure of this paper is as follows. We first describe the empirical data, collecting people's predictions, on which our evaluation of the surprisingly popular method is based, and the benchmark prediction data we use to assess performance. We then analyze the empirical and benchmark data from a number of perspectives, including overall accuracy, the relationship of the surprisingly popular method's predictions to those made by other methods, and the calibration between confidence and accuracy. We discuss insights into the potential applicability of the surprisingly popular method suggested by our findings, and a number of avenues for extending its empirical evaluation as well as improving its ability to make predictions.

\section{Data}

The NFL regular season involves 256 games, with each of 32 teams playing 16 games over a 17-week season, and each team having one bye week. This means each week there are between 13 and 16 games, depending on how many teams have byes that week. 


\section{Experimental Data}

During the 2017-2018 season we collected predictions each week on Wednesday. These predictions were made about every game for that week, which are typically played from Thursday night to the following Monday night. Each week the predictions were made by 100 participants recruited using the Amazon Mechanical Turk (AMT) system, with data collected using the Qualtrics survey system. Different participants were recruited for each week and all were paid US $\$ 1$.

Participants first provided basic demographic information including their gender, and their age range from the options 18-24, 25-34, 35-44, 45-54, 55-64, and $65+$ years. They then rated their knowledge of NFL football on a 5-point scale: extremely knowledgeable, very knowledgeable, moderately knowledgeable, slightly knowledgeable, and not knowledgeable at all. To make predictions about each game, participants first made a prediction about which team they believed would win, then they rated their confidence in this prediction on a 5-point scale: guess, low confidence, moderate confidence, high confidence, and very high confidence. Finally, participants estimated the percentage of others they believed would agree with their prediction. This percentage estimation was done with a slider that showed the exact whole number from 0 to 100 being selected as the slider was moved, as well as permanent indicators $0,10, \ldots, 100$ on a scale.

Once a participant had completed these three behavioral responses - their prediction, confidence rating, and meta-cognitive estimation of agreement — for a game, they pressed an "advance" button to move to the next game. They could adjust the three behavioral responses for a specific game while it was the current game, but could not return to an earlier game once they had advanced. Each participant made predictions about the games in a randomly-determined order.

\section{Benchmark Data}

We also collected benchmark predictions for the 256 games coming from two other sources. One benchmark source was the collation of media expert predictions provided by nflpickwatch.com. These predictions take the form of binary selections for each game. We use the data from 94 experts who provided predictions for all, or almost all, of the 256 games over the season.

The other benchmark data source is provided by the data-science forecasting website fivethirtyeight.com. These predictions are made by an algorithm that is 
a variant ${ }^{1}$ of the Elo statistical method used to measure performance in chess (Elo, 1978). This benchmark is not based on human judgments, but on the history of actual game results for the NFL teams. The Elo predictions are probabilistic and take the form of winning percentages for each team.

\section{Results}

We first analyze the overall accuracy of our AMT participants relative to the media expert benchmark predictions. We find that the sub-group of our participants who self-rated their NFL knowledge as "extremely knowledgeable" have a similar distribution of accuracy to the media experts. Accordingly, we apply the surprisingly popular method, and the other prediction methods based on human judgments, to this sub-group of expert AMT participants. We then compare the effectiveness of these predictions to the media experts and Elo benchmarks, considering first overall accuracy, then patterns of agreement and disagreement between predictions, and finally the calibration of the predictions.

\section{Accuracy by Expertise}

Figure 2 examines the behavior of the AMT participants, in terms of the accuracy of their predictions, the relationship between their confidence ratings and accuracy, and the calibration of their meta-cognitive estimates of agreement.

The left panels show the distribution of accuracy over all 256 games for six different groups. At the top is the distribution for the media experts. Below are the distributions for the AMT participants, divided into the five categories of selfrated knowledge of NFL football. Within each panel, the area of each square is proportional to the number of people with that level of accuracy. The total area of the squares in each distribution is proportional to the number of participants in each category showing, for example, that more AMT participants rated themselves as "very knowledgeable" or "moderately knowledgeable" than "not knowledgeable at all".

The middle panels of Figure 2 show the mean accuracy of predictions made with different levels of confidence, again broken into the self-rated knowledge groups. Thus, for example, the top-most panel in the middle column shows how accurate

\footnotetext{
${ }^{1} \mathrm{~A}$ set of FAQ answers at https://fivethirtyeight.com/features/introducing-nfl-elo -ratings/ provide more details.
} 


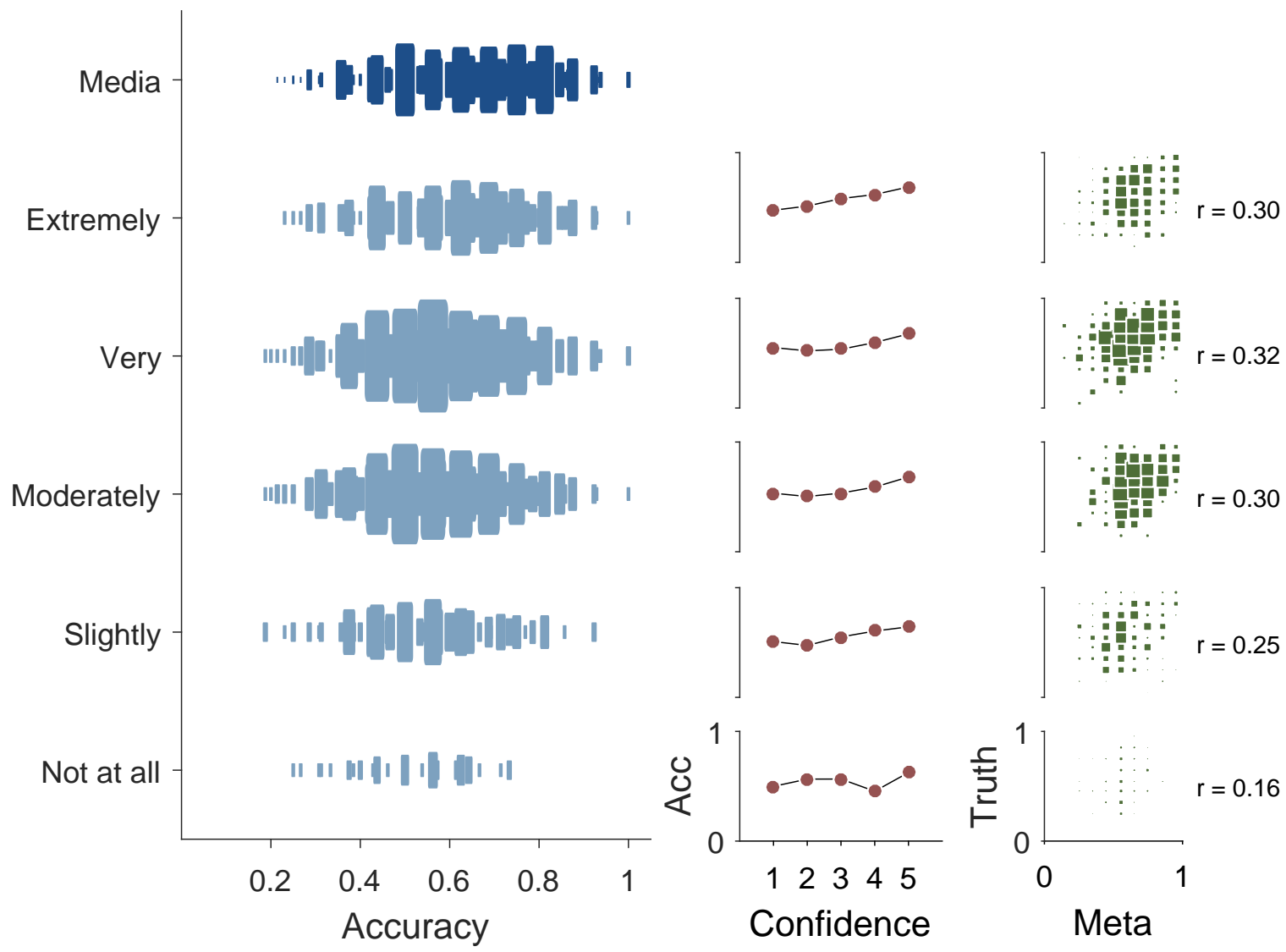

Figure 2. The left panel shows the distribution of prediction accuracy for six different groups of people. At the top is the distribution for media experts and below are the distributions for AMT participants with different levels of self-rated overall knowledge. The middle panels shows the relationship between accuracy and confidence ratings for each selfrated knowledge group. The right panels show the relationship between the meta-cognitive estimate and true level of agreement in each prediction for each self-rated knowledge group.

decisions made with confidence ratings of $1, \ldots, 5$ are on average, for just those AMT participants with the highest self-knowledge rating. These results show that, in general, more confident predictions were more accurate for all of the knowledge levels. The calibration of confidence and accuracy is most impressive for the "extremely knowledgeable" group. It is nearly linear, and has no departures from monotonicity.

The right panels of Figure 2 show the relationship between the meta-cognitive estimate of agreement and the true level of agreement, again broken into the selfrated knowledge groups. The correlations between the estimate and the truth are 
around 0.3 for all of the groups, except for the "not knowledgeable at all" group, which performs more poorly.

Collectively, the results in Figure 2 led us to focus on the group of AMT participants who rated themselves as "extremely knowledgeable". The left panels show that this group makes predictions with levels of accuracy that are most similar to those of the media experts. ${ }^{2}$ The middle panels show that they also supply meaningful confidence ratings for their individual decisions, with a monotonic relationship between increased confidence and increased accuracy. The right panels show that, while none of the groups is especially good at making these judgments, the "extremely knowledgeable" group is at least as good as the others.

Given the goal of testing the ability of the surprisingly popular method to make accurate predictions, it makes sense to focus on those participants who are evidently supplying good predictions, calibrated confidence judgments, and reasonable meta-cognitive estimates. Thus, the following results focus exclusively on the AMT participants who self-rated as "extremely knowledgeable" about the NFL. The ideal experimental test of the surprisingly popular method would have been to ask the media experts to provide meta-cognitive judgments. Obviously, this was not possible, and so the group of AMT participants who most closely match the media experts in terms of prediction accuracy serves as a sensible surrogate group.

It is worth noting that the basis for identifying this group is self-rating data that was available before the games were played, and so could form part of a real-world prediction method. We acknowledge, however, that there is an exploratory aspect to this method of analysis. In particular, although we posted all of our raw data before games were played, the analysis method was not pre-registered. Thus, it is important to replicate our findings to test the efficacy of self-rating in identifying accurate participants. For completeness, in the supplementary information, we present analyses of the surprisingly popular method based on all of the AMT participants. The pre-

\footnotetext{
${ }^{2}$ Bayes factors for independent samples $t$-tests (Rouder, Speckman, Sun, Morey, \& Iverson, 2009) provided evidence that the media experts were more accurate than each of the AMT participant groups. This evidence of difference was overwhelming for the very, moderately, slightly, and not at all confident groups, with the log Bayes factors of 36, 50, 35, and 13 in favor of a difference, respectively. The evidence was much more equivocal for the comparison with the expert group of AMT participants, with a $\log$ Bayes factor of 2.9. Thus, while the balance of evidence is that the media experts were also more accurate than the self-rated "extremely knowledgeable" participants, this is clearly the closest-matching group.
} 


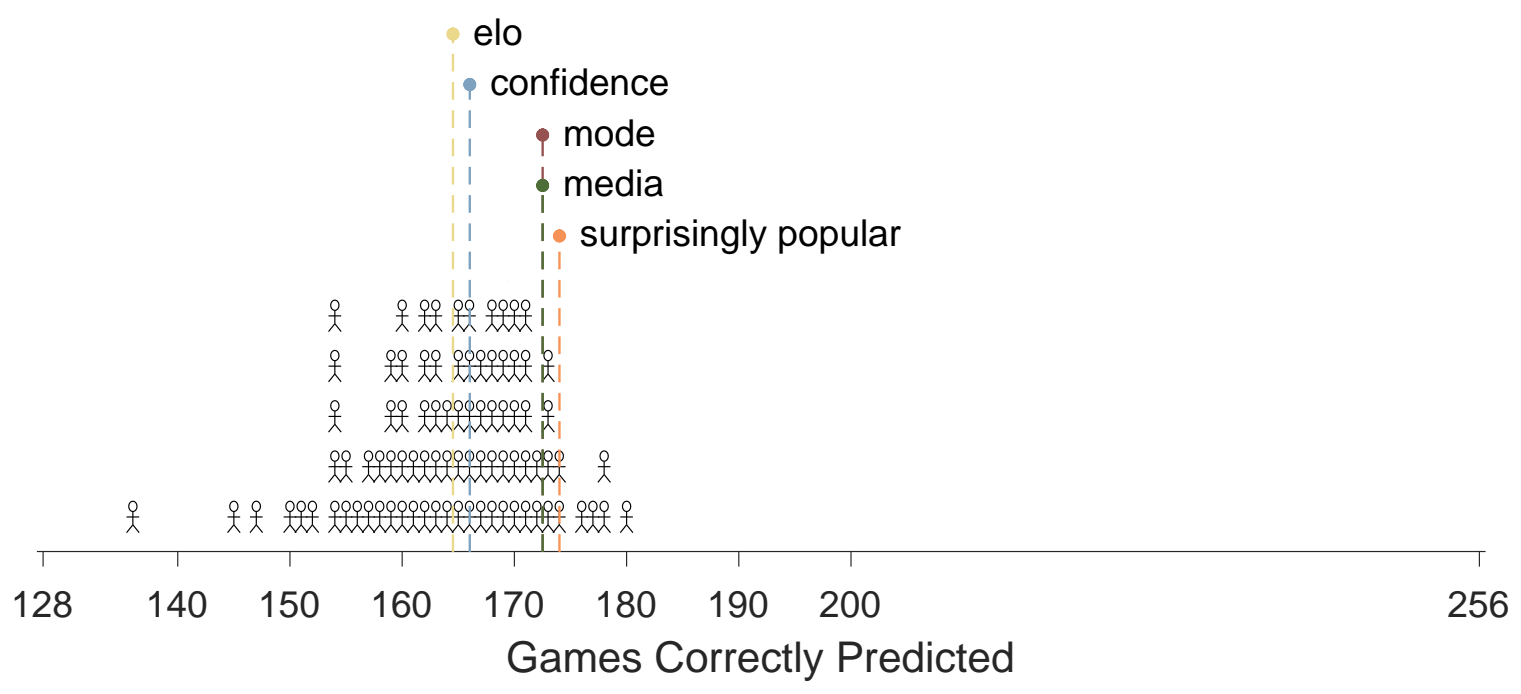

Figure 3. The number of games correctly predicted by the surprising popular (sp), confidence-weighted tally (conf), mode of AMT participants (mode), mode of media experts (media), and Elo (elo) methods, and for 94 individual media experts. The stick figures represent the distribution of the number of games correctly predicted by the media experts. The labeled lines show the number of games correctly predicted by the methods.

diction accuracy achieved by using all of the participants turns out to be much less impressive.

Accuracy of the Surprisingly Popular Method and Other Predictions

Figure 3 summarizes the overall performance of the surprisingly popular method, in the context of the performance of the individual media experts and other comparison methods. The stick figures show the distribution of the total number of games in the season correctly predicted by the 94 individual media experts. The worst-performed experts correctly predicted the outcome of around 150 of the 256 games, the best-performed experts correctly predicted about 180 games, and most experts were correct for between 155 and 175 of the games.

The surprisingly popular method, based on the group of AMT participants selfrated as "extremely knowledgeable", predicted 174 games correctly. This performance was inferior to five of the media experts, the same as two more, and superior to the remaining 87 . The surprisingly popular method also slightly outperformed the modal (most common) predictions of the "extremely knowledgeable" AMT participants and 
the modal predictions of the media experts. These mode-based predictions were both correct for 172 of the 256 games. The confidence-weighted tally of the "extremely knowledgeable" AMT made correct predictions for 162 games. These predictions were based on the team with the larger sum of confidence ratings across all of the participants favoring that team. The team with the greater Elo predicted winning percentage was correct for 162 games. $^{3}$

\section{Examples of the Surprisingly Popular Method}

Figure 4 presents three examples of the performance of the surprisingly popular method, and the confidence-weighted tally method, aimed at giving some insight into the relative success of the surprisingly popular method. The detailed results for every game are available in the supplementary material. The three examples in Figure 4 were chosen because the surprisingly popular and confidence-weighted tally methods make different predictions and because, in two cases, the surprisingly popular prediction does not follow the majority. It is the ability of the surprisingly popular method to predict against confidence-based and majority opinion that makes it theoretically interesting.

For each of the games in Figure 4 - between the Green Bay Packers and Seattle Seahawks in week 1, the Denver Broncos and Dallas Cowboys in week 2, and the Seattle Seahawks and Philadelphia Eagles in week 15 - the surprisingly popular method is shown on the left, and the confidence-weighted tally method is shown on the right. The surprisingly popular method uses the same display format used in Figure 1. The confidence-weighted tally method is shown by histograms representing the distribution of confidence ratings on the five-point scale. The confidence distribution for predictions favoring the first-named team is above and the confidence distribution for predictions favoring the second-named team is below. The tally of these confidence ratings is displayed as is the prediction made by the method.

In the Packers versus Seahawks game, the predictions of the participants are evenly split between the two teams. The surprisingly popular method predicts a Packers victory because many participants favoring the Packers believe relatively few

\footnotetext{
${ }^{3}$ While the individual media experts made forced choices between the two teams in a game, the aggregate methods can all produce outcomes that favor neither team. The surprisingly popular method can produce equal expert and observed percentages, the confidence-weighted tallies can be equal for both teams, the number of people favoring each team can be equal in determining a mode, and the Elo method can give a $50 \%$ winning percentage to both teams. In these cases, the accuracy of the method was measured as half a game.
} 
Green Bay Packers

Seattle Seahawks

Observed: 50

Expected: 44

Prediction: Packers
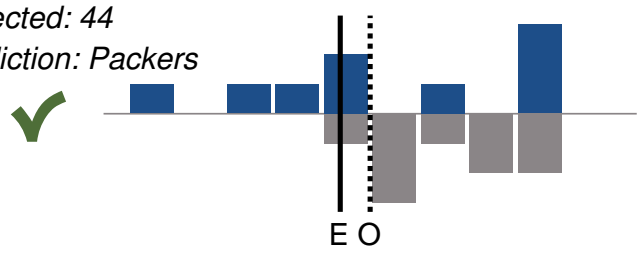

Denver Broncos

Dallas Cowboys

Observed: 53

Expected: 63

Prediction: Cowboys
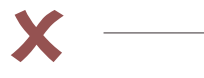

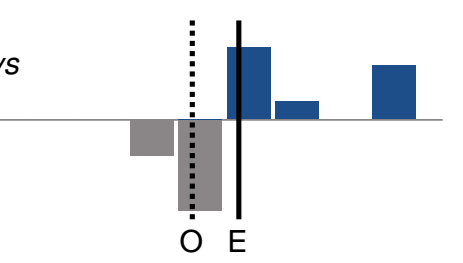

Seattle Seahawks

Philadelphia Eagles

Observed: 42

Expected: 36

Prediction: Seahawks

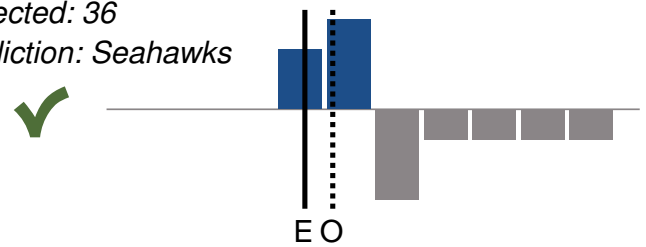

Green Bay Packers

Seattle Seahawks

Packers: 26

Seahawks: 30

Prediction: Seahawks

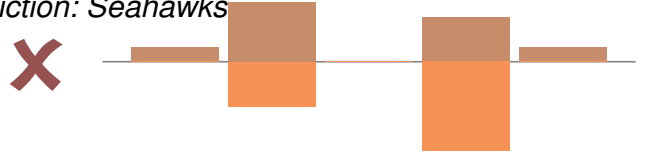

Denver Broncos

Dallas Cowboys

Broncos: 31

Cowboys: 12

Prediction: Broncos
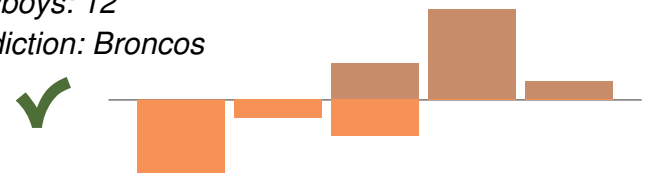

Seattle Seahawks

Philadelphia Eagles

Seahawks: 13

Eagles: 28

Prediction: Eagles

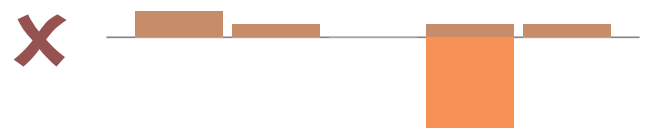

Figure 4. Illustrative examples of the surprisingly popular and confidence-weighted tally methods for three NFL games.

others will agree with them. This makes the relatively high percentage of participants predicting a Packers above the expectation. The confidence-weighted tally method, however, predicts a Seahawks victory, because participants favoring the Seahawks tended to do so with higher confidence. This game provides a good example of the different mechanisms by which these two methods break a 50-50 tie in the binary predictions. As the tick and cross marks in Figure 4 indicate, the winner of the game was the Packers, and so the surprisingly popular method made the correct prediction.

In the Broncos versus Cowboys game, the surprisingly popular method chooses against the majority prediction. A total of $53 \%$ of participants favored the Broncos, but many participants favoring the Cowboys expected relatively few others to agree 
with them, leading to a higher expectation of $63 \%$. Thus, the observed percentage in favor of the Broncos fell short of the expectation, and the "underdog" Cowboys were predicted. Meanwhile, the confidence-weighted tally method predicted a Broncos victory by some margin, since participants favoring the Broncos generally expressed high confidence while participants favoring the Cowboys generally expressed low confidence. As it turned out, the Cowboys won the game, so the confidence-weighted tally method made the correct prediction.

Finally, in the Seahawks versus Eagles game, there is an expectation that the Eagles are heavy favorites. Most participants predicting an Eagles win believe the majority of others will agree with them. Most participants predicting a Seahawks win believe the majority will disagree with them and favor the Eagles. Thus, even though a minority of $42 \%$ of participants predicted the Seahawks would win, this is higher than than the $26 \%$ expectation, and they were correctly predicted to win by the surprisingly popular method.

\section{Overlap in Predictions}

Figure 5 provides a summary of the relationship between the predictions of the different methods and their accuracies. The panels correspond to all 10 possible pairings of the five aggregation methods. Within each panel, a $2 \times 2$ table is shown counting how often the pair of methods both made correct predictions, both made incorrect predictions, or one method was correct and the other was not. The left column corresponds to correct predictions for the method labeled at the top, and the top row corresponds to correct predictions for the method labeled at the left. Within each cell, the area of the square is proportional to how often the pattern of correct and incorrect predictions across the pair of methods was observed, and the actual count is also displayed.

The results in Figure 5 show that the surprisingly popular method and the confidence-weighted tally method tend to produce more similar predictions than other pairings of methods. The two mode-based methods using expert judgments, for the AMT participants and the media experts, are also quite similar in their predictions. Meanwhile, the surprisingly popular method has the least overlap in its predictions with those made by other data sources: the media experts and the Elo method. Collectively, these results suggest that both the data source and the aggregation method contribute to the patterns of predictions. 

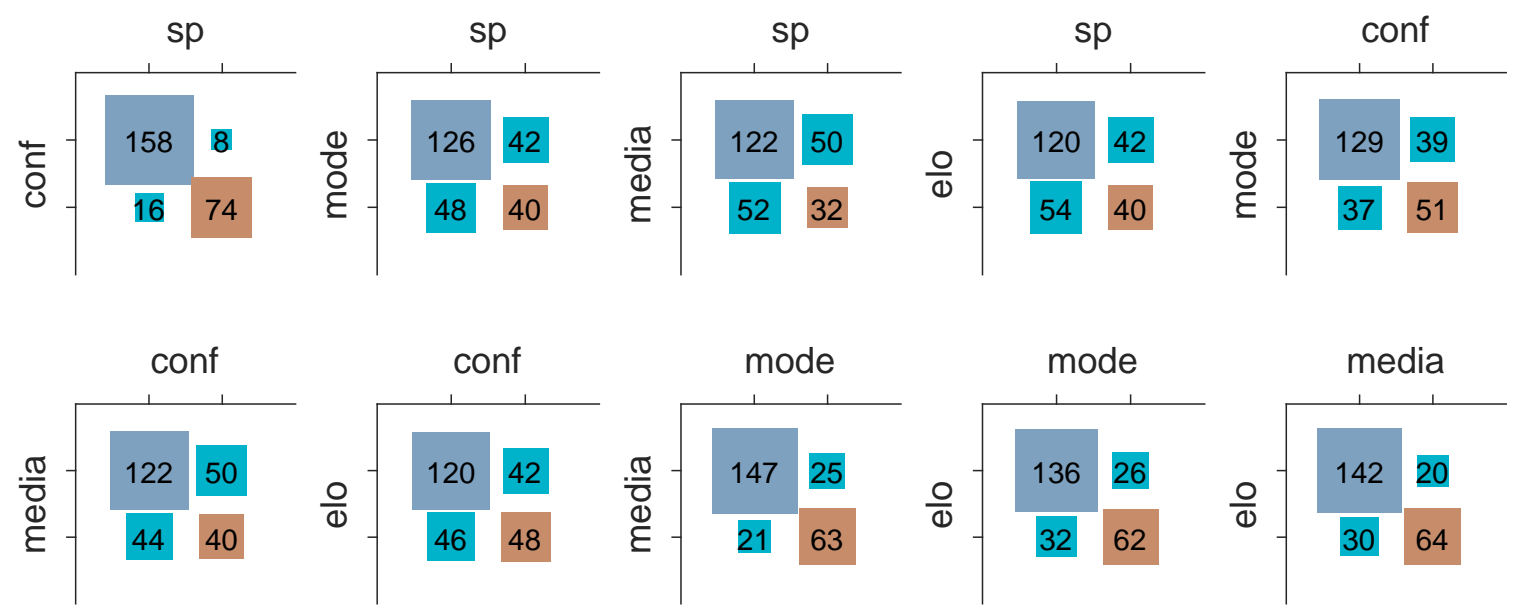

Figure 5. Relationship between pairs of methods and the accuracy of their predictions. Each panel corresponds to one of the 10 unique pairings of the five methods: surprising popular (sp), confidence-weighted tally (conf), mode of AMT participants (mode), mode of media experts (media), and Elo (elo). Within each panel, the areas of squares and overlain numbers show counts of games in which both methods made the same correct prediction (top-left), the same incorrect prediction (bottom-right), the left-labeled row method made a correct prediction but the top-labeled column method did not (top-right), or the toplabeled column method made a correct prediction but the left-labeled row method did not (bottom-left)

\section{Calibration}

Our final analysis considers the calibration of predictions made by the surprisingly popular and other methods. Ideally, a method would always make correct predictions, but, since errors are inevitable, it is important to know how much confidence should be placed in a prediction. Confidence guides decisions and actions that might be made based on the predictions, such as betting. We examine the calibration of each method in terms of a calibration curve that relates the confidence in predictions to their empirical accuracy (Lichtenstein, Fischoff, \& Phillips, 1982).

For the Elo method, the confidence in a decision is given directly by the winning probabilities it produces. For the methods based on the majority prediction across people, the proportion of people in the majority is a natural measure of confidence (Grofman, Owen, \& Feld, 1983; Kerr \& Tindale, 2004). For the confidence-weighed tally method, an obvious measure of confidence is the tally in favor of the team predicted to win, expressed a proportion of the sum of both tallies. as a difference 


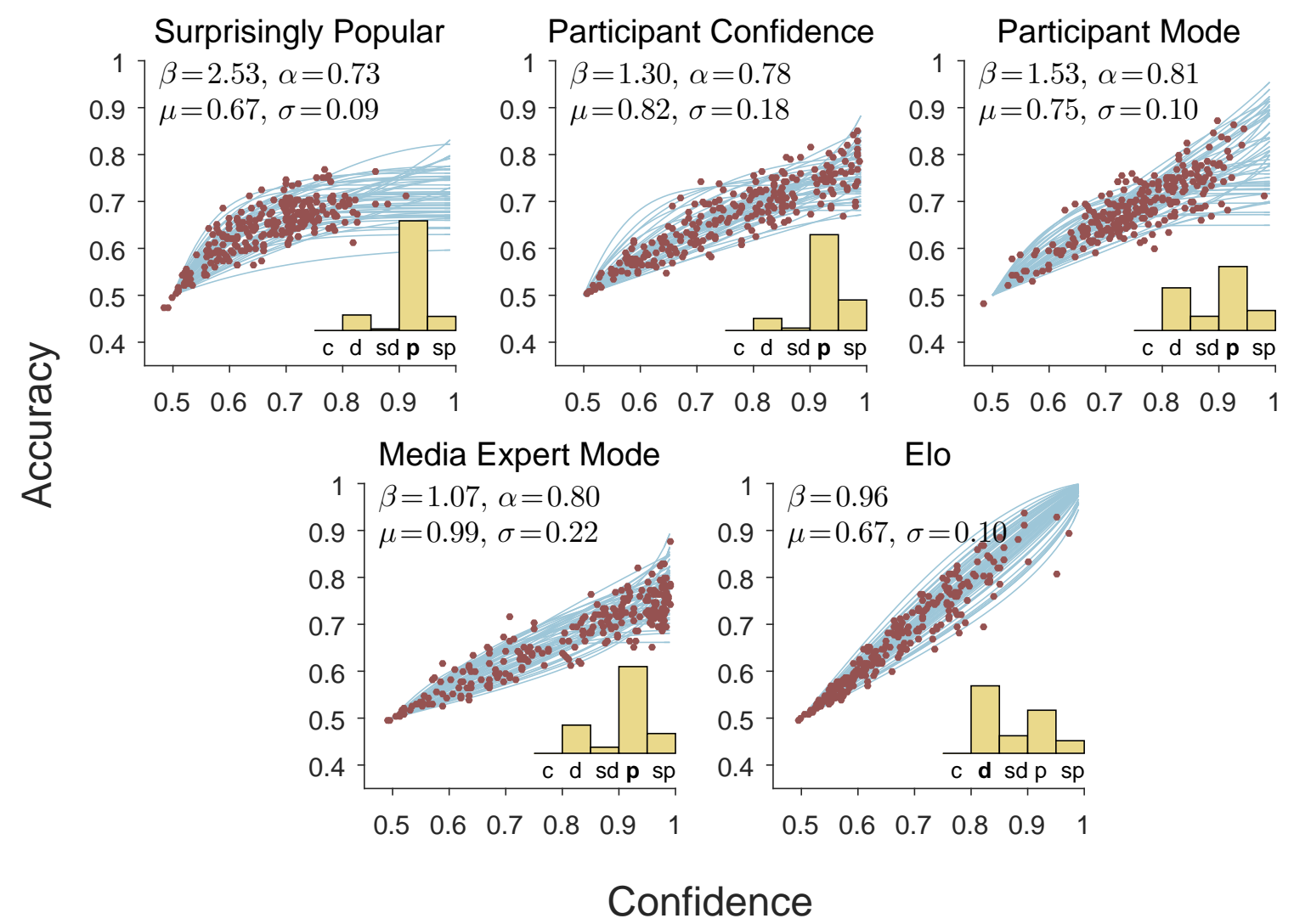

Figure 6. Results of the calibration analysis for the five aggregation methods. In each panel, the inset histogram shows the posterior probability of the 5 possible logistic growth models ("c" = chance, "d" = deterministic, "sd" = shifted deterministic, "p" = probabilistic, "sp" = shifted probabilistic) used by Lee \& Lee (2017). The most likely model is labeled in bold. The lines show samples from the posterior distribution of the most likely calibration model, and the circular markers show samples from the joint distribution of confidence and accuracy for the predictions of the NFL games.

between the confidence tallies. For example, in the Houston Texans versus Tennessee Titans game in the top-right of Figure 4, the Titans are predicted to win, since their tally of 27 is greater than 22 . The confidence in this prediction, according to the measure we use, is $27 /(27+22) \approx 55 \%$. For the surprisingly popular method, one possible way to measure confidence is the difference between the expected and observed percentages for a team, since it is their difference that determines the prediction. We add this difference to the chance probability of $50 \%$ to determine the confidence the surprisingly popular method has in each prediction it makes. For example, for the same Houston Texans versus Tennessee Titans game, now in the top-left of Figure 4, 
the Texans are predicted to win, since the observed percentage of people predicting them to win is $47 \%$, which is greater than the expected $45 \%$. The confidence in this prediction, according to the measure we use, is $50+|47-45|=52 \%$.

To examine the calibration relationship between confidence and accuracy, we use the statistical approach developed by Lee and Lee (2017), which is summarized in the supplementary information. This approach infers calibration curves based on logistic growth functions. It aims first to infer what type of growth function best describes the calibration curve and then infers the parameters of the appropriate function. Both the type of curve and the parameters have meaningful psychological interpretations, which help to characterize and measure the calibration properties of a method. Figure 6 shows the results of the calibration analysis for the five methods. For each method, the inferred type of calibration curve is shown by the inset histogram. The curve relates the confidence a method has in a prediction on the $x$-axis to the probability the prediction is correct on the $y$-axis. The inferred posterior distribution for the calibration curve is shown by the lines, with the posterior for individual predictions shown by circular markers.

The inferred parameters from the Lee and Lee (2017) analysis for each method are also shown in Figure 6. The parameter $\beta$ measures how quickly additional confidence leads to more accurate predictions (i.e., the slope of the calibration curve); $\alpha$ measures the upper-bound on accuracy (i.e., how accurate decisions made with $100 \%$ confidence are expected to be); $\mu$ measures the mean level of confidence a method has across all of its predictions; and $\sigma$ measures the standard deviation of the distribution of confidence. The Elo method stands out as being extremely well calibrated. It has a slope near $\beta=1$. It is inferred to be deterministic, meaning that in theory a $100 \%$ confident prediction will be $100 \%$ accurate. The mean level of confidence is about $67 \%$ and rarely are predictions made with more than $80 \%$ certainty. This caution contrasts with the high confidence of the media modal decision, which has a mean confidence of $\mu=0.99$ but has an upper-bound on accurate of $\alpha=0.80$. The participant mode and confidence-weighted tally calibration curves fall somewhere between these extremes. Both show a broader distribution of confidence. They have similar upper bounds on accuracy to the media mode method.

It does not make sense to compare the calibration curve of the surprisingly popular method to the calibration curves of the other methods, even though it is our focus. While the difference between expected and observed proportions is the 
obvious way to generate a measure of confidence, it is not obvious how this difference should be scaled. The current scaling assumes a difference of $50 \%$ is maximal, which seems reasonable, but not the only possible choice. In addition, it is not obvious that the scaling should be linear, as is currently assumed. Theoretical development is needed to provide a strong test of the calibration properties of the surprisingly popular method. What the results in Figure 6 do suggest, however, is that the surprisingly popular method is at least capable of reasonable calibration, in the sense that increasing differences between the observed and expected agreement correspond to more accurate decisions.

\section{Discussion}

Our results suggest there is promise in applying the surprisingly popular method to predicting the outcomes of NFL games. The surprisingly popular method outperformed most of the media experts, the majority opinion of these experts, the Elo method, and other measures based on our AMT participant data. While the improvement in accuracy was small, the nflpickwatch.com and Elo measures provide genuine real-world benchmarks. If the current level of improvement was replicated for other seasons, it would be have implications for real-world prediction and betting.

Despite this promise, we recognize the limitations of evaluating of any prediction method based on only one season, comprising a few hundred binary outcomes. Accordingly, we view this study as a motivating demonstration of the applicability of the surprisingly popular method to making predictions, with a particular focus on the important class of predictions represented by sporting contests. It seems clear that people were able to make decisions and provide meta-cognitive judgments in a prediction setting as naturally as they are able in previously studied non-prediction settings like general knowledge questions. Indeed, as the examples in Figure 4 highlight, the basic insight of the surprisingly popular method can generalize to predictive settings. If a subset of people have insight into a surprise winner, the surprisingly popular method provides a simple and effective way to capture and use that knowledge.

The key question, therefore, is whether and how often such subsets of people exist. Our study shows that they can exist and suggests that domain knowledge, as measured by self-rating in our case, may be an important factor. Future work should try and isolate the type of expertise, or the types of games, that are likely to have the private knowledge or insight needed. As we mentioned, the ideal test is how well the 
surprisingly popular method performs when media experts provide meta-cognitive estimates of agreement, without being aware of the predictions others are making.

Another direction for future research is suggested by the meta-cognitive judgments that are the novel empirical feature of the surprisingly popular method. It is clear from the illustrative examples in Figure 4 that the distributions of these metacognitive estimates are generally broad. Whether this arises because of a individual differences in opinion about the percentage, individual differences in the cognitive processes in the way in the estimates are generated, or both, is an interesting cognitive modeling question. Potentially, a model-based account of these data could be incorporated into the surprisingly popular method to improve the precision of the meta-cognitive estimates (McCoy \& Prelec, 2017). Ideally, differences due to the cognitive processes involved in meta-cognition could be "factored out", leaving the key signal of expected agreement.

The surprisingly popular method is a clever and simple approach to combining people's judgments to make accurate good group decisions, based on the insight that meta-cognitive information is important. Our results suggest that it may be useful in forecasting applications involving genuine predictions, as an alternative or complementary method to more elaborate approaches like prediction markets (Silver, 2012). We hope that our results encourage the further investigation of the surprisingly popular method, both in terms of its real-world performance and the cognitive modeling challenges it poses for understanding the relationship between choice and meta-cognition.

\section{Acknowledgments}

A project page for this paper on the Open Science Framework project page at https://osf.io/3kjmu/ includes all of the raw data from our NFL data collection, code for the calibration modeling analyses, the results of the surprisingly popular method applied to all 256 games, and a screen shot of the experimental interface. We thank Drazen Prelec and colleagues for supplying the raw data from Prelec et al. (2017).

\section{References}

Elo, A. E. (1978). The rating of chessplayers, past and present. Arco. 
Grofman, B., Owen, G., \& Feld, S. L. (1983). Thirteen theorems in search of the truth. Theory and Decision, 15, 261-278.

Kerr, N. L., \& Tindale, R. S. (2004). Group performance and decision making. Annual Review of Psychology, 55, 623-655.

Lee, M. D., \& Lee, M. N. (2017). The relationship between crowd majority and accuracy for binary decisions. Judgment and Decision Making, 12, 328-343.

Lichtenstein, S., Fischoff, B., \& Phillips, L. D. (1982). Calibration of probabilities: The state of the art to 1980. In D. Kahneman, P. Slovic, \& A. Tversky (Eds.), Judgment under uncertainty: Heuristics and biases (pp. 306-334). Cambridge, England: Cambridge University Press.

McCoy, J., \& Prelec, D. (2017). A statistical model for aggregating judgments by incorporating peer predictions. arXiv preprint arXiv:1703.04778.

Plummer, M. (2003). JAGS: A program for analysis of Bayesian graphical models using Gibbs sampling. In K. Hornik, F. Leisch, \& A. Zeileis (Eds.), Proceedings of the 3rd International Workshop on Distributed Statistical Computing (DSC 2003).

Prelec, D., Seung, H. S., \& McCoy, J. (2017). A solution to the single-question crowd wisdom problem. Nature, 541, 532-535.

Rouder, J. N., Speckman, P. L., Sun, D., Morey, R. D., \& Iverson, G. (2009). Bayesian ttests for accepting and rejecting the null hypothesis. Psychonomic Bulletin $\mathscr{G}$ Review, $16,225-237$.

Silver, N. (2012). The signal and the noise: Why so many predictions fail - but some don't. Penguin.

Tetlock, P. E., \& Gardner, D. (2016). Superforecasting: The art and science of prediction. Random House. 


\section{Supplementary Information}

Results For All AMT Participants

Our application of the surprisingly popular method relies on just those AMT participants who self-rated as being "extremely knowledgeable" about NFL football. The rationale for limiting the analysis to these participants was to use them as surrogate experts, from whom the meta-cognitive estimation of agreement was available. It is natural, however, also to consider the performance of the surprisingly popular method based on all of the available AMT participants. These results are summarized in FIgure 7. It is clear the surprisingly popular method performs much more poorly, with an accuracy well below the mode of the media experts, and in the middle of the distribution of the individual accuracies for these experts. Presumably, this decrease in accuracy is the result of the less accurate judgments made by the non-expert AMT participants, as evident in the left-hand panels of Figure 2.

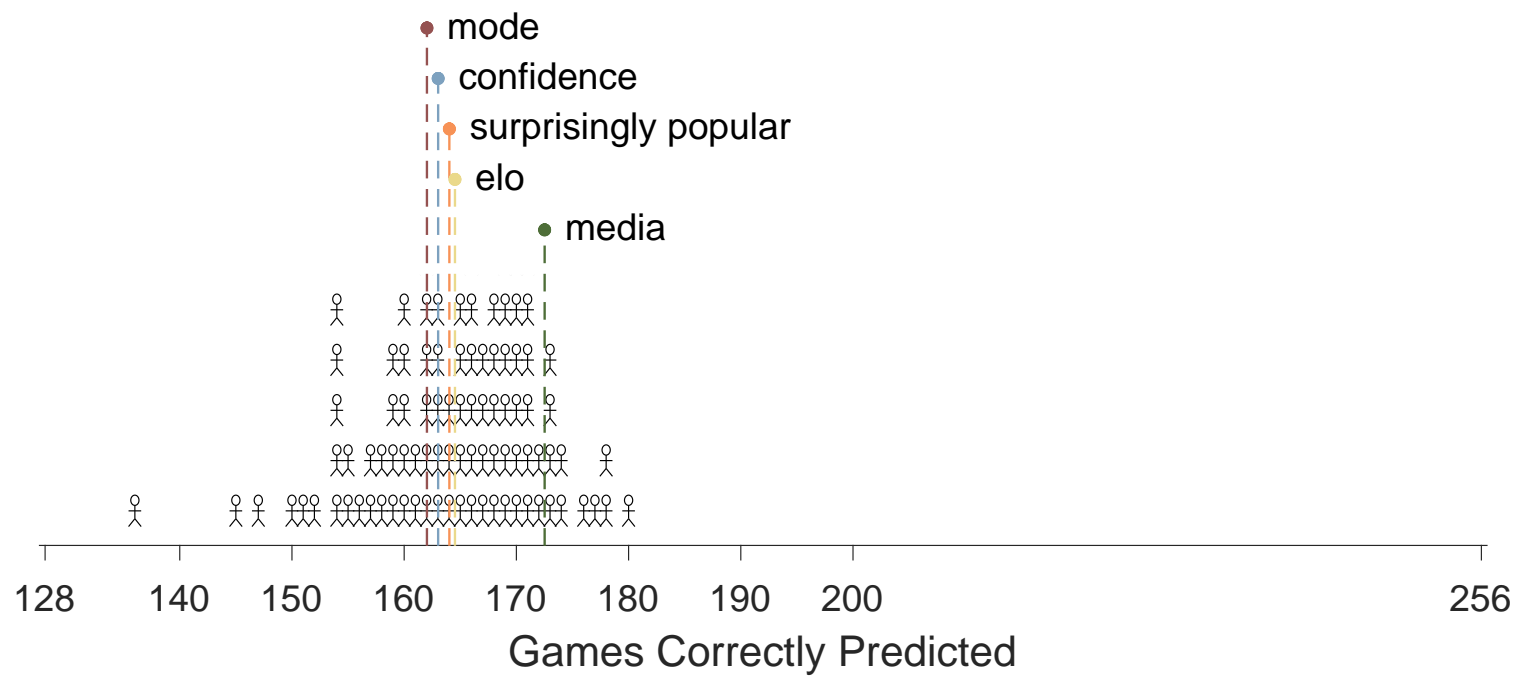

Figure 7. The number of games correctly predicted by the surprising popular (sp) , confidence-weighted tally (conf), mode of AMT participants (mode), based on all of the AMT participants. The number of games correctly predicted by the mode of media experts (media) and Elo (elo) methods, and for 94 individual media experts, are shown. 

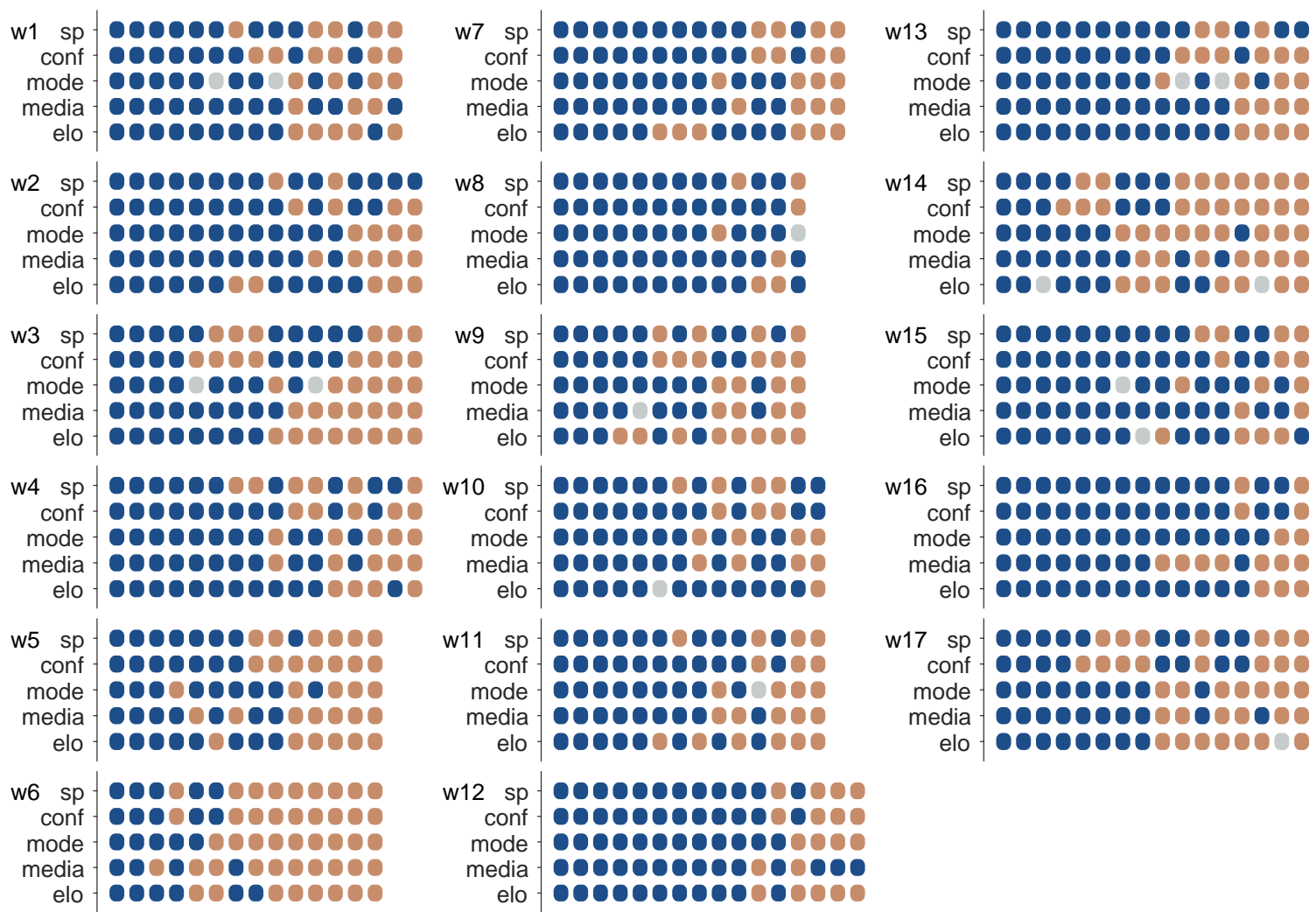

Figure 8. The accuracy of the predictions made by the surprising popular (sp), confidenceweighted tally (conf), mode of AMT participants (mode), mode of media experts (media), and Elo (elo) methods, for every game of the NFL season. Panels correspond to weeks of the seasons, rows to methods, and columns to games. Dark blue circles indicate a correct prediction; light orange circles indicate an incorrect prediction; and grey circles indicate neither team was favored.

\section{All Predictions}

Figure 8 details the accuracy of every aggregate prediction method for every game. Each panel corresponds to a week, labeled "w1" for week 1, and so on. Within each panel, rows correspond to methods, and columns to games, ordered from leftto-right from best overall predicted to worst overall predicted. A dark blue circle indicates a correct prediction; a light orange circle indicates an incorrect prediction; a gray circle indicates neither team was favored by the method. Not surprisingly, there is often strong agreement across the methods. Most weeks include a number of games where all the methods make the same prediction: more often than not this 
prediction is correct, leading to a column of blue circles, but sometimes it is incorrect, leading to a column of orange circles.

\section{Calibration Modeling}

Our calibration analysis is a natural extension of the approach developed by Lee and Lee (2017). Their approach applies directly only to the prediction methods based on the majority decision over a set of individuals, but can be generalized straightforwardly to include methods with other measures of decision confidence. Formally, in the Lee and Lee (2017) approach, there are $n$ decisions, and the $i$ th decision has $k_{i}$ people making the majority decision out of $n_{i}$ people, with an accuracy of $y_{i}=1$ if the majority decision is correct, and $y_{i}=0$ otherwise. The proportion $\theta_{i}$ represents the size of the majority and the probability $\phi_{i}$ representing the probability the decision correct.

This approach applies directly to the majority decisions based on AMT participants and media experts. The observed majority is assumed follows a binomial distribution with respect to the underlying majority proportion and the total number of people, so that $k_{i} \sim \operatorname{Binomial}\left(\theta_{i}, n_{i}\right)$. For the surprisingly popular, confidenceweighted tally, and Elo methods, which produce a continuous measure of confidence, $c_{i}$ for the $i$ th decision, we assume this observed confidence reflects the underlying confidence with a small amount of noise, so that $c_{i} \sim \operatorname{Gaussian}\left(\theta_{i}, 1 /(0.01)^{2}\right)$. In both cases, the observed accuracy is a Bernoulli draw with respect to the underlying accuracy, $y_{i} \sim \operatorname{Bernoulli}\left(\phi_{i}\right)$ and the distribution of confidence across all decisions is modeled as coming from an over-arching truncated Gaussian distribution, so that $\theta_{i} \sim \operatorname{Gaussian}_{(0,1)}\left(\mu, 1 / \sigma^{2}\right)$ where $\mu \sim \operatorname{Uniform}\left(\frac{1}{2}, 1\right)$ and $\sigma \sim \operatorname{Uniform}\left(0, \frac{1}{2}\right)$ are the mean and standard deviation, respectively.

To model the relationship between confidence and accuracy, Lee and Lee (2017) rely on a logistic growth model of the form

$$
\phi_{i}=\alpha /\left(1+\exp \left\{-\beta\left[\log \frac{\theta_{i}}{1-\theta_{i}}-\log \frac{\delta+\frac{1}{2}}{1-\left(\delta+\frac{1}{2}\right)}-\frac{1}{\beta} \log (2 \alpha-1)\right]\right\}\right),
$$

with a growth parameter $\beta$, a bound parameter $\alpha$, and a shift parameter $\delta$. This model is shown in the central panel of Figure 9. The upper bound on accuracy is controlled by $\alpha$. As the confidence in a decision increases, its accuracy increases at a rate controlled by $\beta$, with $\beta=1$ corresponding to a linear increase, values $\beta>1$ 

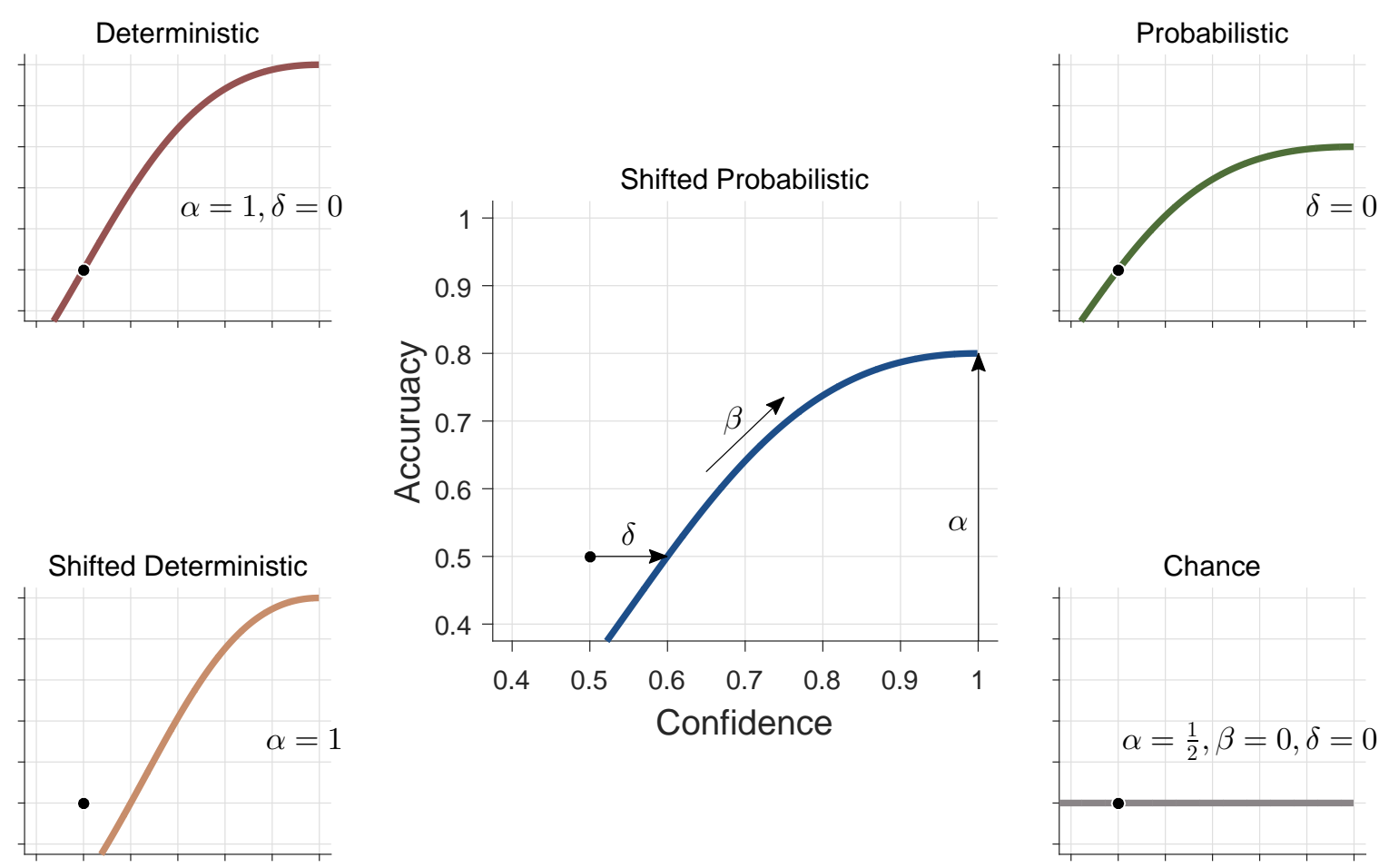

Figure 9. Logistic growth calibration curves relating confidence on the $x$-axis to accuracy on the $y$-axis. The central panel shows the general model, with a growth parameter $\beta$, a bound parameter $\alpha$, and a shift parameter $\delta$. Surrounding panels show specific-case nested models with natural interpretations. Based on Lee \& Lee (2017, Figure 3).

corresponding to faster increases, and values $\beta<1$ corresponding to slower increases. In the limit $\beta=0$, there is no growth in accuracy. The shift of the growth curve is controlled by $\delta$. When $\delta=0$ a decision made with no confidence has chance accuracy.

Lee and Lee (2017) also consider four special cases of the full shifted probabilistic model, shown in the surrounding panels of Figure 9. The deterministic model in the top-left corresponds to setting $\delta=0, \alpha=1$, and allowing only $\beta$ to vary. The deterministic shift model in the bottom-left corresponds to setting just $\alpha=1$, allowing for worse-than-chance performance. The probabilistic model in the top-right corresponds sets $\delta=0$ so that accuracy starts at chance and grows to some upper bound $\alpha$ as confidence increases. Conceptually, $\alpha$ corresponds to the upper bound measuring the inherent (un)predictability of the domain, and $\beta$ corresponds to how quickly that limit is approached. Finally, the chance model in the bottom-right cor- 
responds to setting $\alpha=\frac{1}{2}, \beta=0$, and $\delta=0$, which reduces the model to $\theta=\frac{1}{2}$ for all levels of confidence, so that accuracy is always at chance.

We follow Lee and Lee (2017) by implementing this model as a graphical model in JAGS (Plummer, 2003), which allows for fully Bayesian inference using computational sampling methods. The full shifted probabilistic model, and its four special cases, are treated as components of a latent-mixture model, allowing both the type of calibration curve and its parameters to be inferred. 